\title{
Photoionization cross section by Stieltjes imaging applied to coupled cluster Lanczos pseudo-spectra
}

Janusz Cukras, Sonia Coriani, Piero Decleva, Ove Christiansen and Patrick Norman

\section{Linköping University Post Print}

\section{Tweet}

N.B.: When citing this work, cite the original article.

Original Publication:

Janusz Cukras, Sonia Coriani, Piero Decleva, Ove Christiansen and Patrick Norman, Photoionization cross section by Stieltjes imaging applied to coupled cluster Lanczos pseudospectra, 2013, Journal of Chemical Physics, (139), 9.

http://dx.doi.org/10.1063/1.4819126

Copyright: American Institute of Physics (AIP) http://www.aip.org/

Postprint available at: Linköping University Electronic Press http://urn.kb.se/resolve?urn=urn:nbn:se:liu:diva-100318 


\title{
Photoionization cross section by Stieltjes imaging applied to coupled cluster Lanczos pseudo-spectra
}

\author{
Janusz Cukras, ${ }^{1}$ Sonia Coriani, ${ }^{1}{ }^{1, a)}$ Piero Decleva, ${ }^{1}$ Ove Christiansen, ${ }^{2}$ \\ and Patrick Norman ${ }^{3}$ \\ ${ }^{1}$ Dipartimento di Scienze Chimiche e Farmaceutiche, Università degli Studi di Trieste, via L. Giorgieri 1, \\ I-34127 Trieste, Italy \\ ${ }^{2}$ Department of Chemistry, Aarhus University, DK-8000 Aarhus C, Denmark \\ ${ }^{3}$ Department of Physics, Chemistry and Biology, Linköping University, SE-581 83 Linköping, Sweden
}

(Received 26 May 2013; accepted 9 August 2013; published online 3 September 2013)

\begin{abstract}
A recently implemented asymmetric Lanczos algorithm for computing (complex) linear response functions within the coupled cluster singles (CCS), coupled cluster singles and iterative approximate doubles (CC2), and coupled cluster singles and doubles (CCSD) is coupled to a Stieltjes imaging technique in order to describe the photoionization cross section of atoms and molecules, in the spirit of a similar procedure recently proposed by Averbukh and co-workers within the Algebraic Diagrammatic Construction approach. Pilot results are reported for the atoms $\mathrm{He}, \mathrm{Ne}$, and $\mathrm{Ar}$ and for the molecules $\mathrm{H}_{2}, \mathrm{H}_{2} \mathrm{O}, \mathrm{NH}_{3}, \mathrm{HF}, \mathrm{CO}$, and $\mathrm{CO}_{2}$. (C) 2013 AIP Publishing LLC. [http://dx.doi.org/10.1063/1.4819126]
\end{abstract}

\section{INTRODUCTION}

With the advent of third generation synchrotron radiation facilities, Free Electron Lasers, and High Harmonic Generation, coupled to high resolution and multicoincidence detectors, research on photoionization phenomena has been particularly stimulated. This has resulted in a large number of experimental data for the spectroscopic parameters describing such phenomena, like total and differential cross-sections for all the involved channels. Besides their fundamental importance as a basic probe of structural and dynamical properties of many-body systems, molecular photoionization processes are basic ingredients in a variety of contexts, e.g., astrophysics, aeronomy, radiation chemistry, environmental and atmospheric chemistry, metrology, surface science and catalysis, and new material development. ${ }^{1-5}$

Photoionization cross sections are used, for instance, in the determination of the ionization structure of cosmic gas subjected to ultraviolet and X-ray radiation. ${ }^{1,2}$ Accurate absolute photo-absorption and total photoionization cross sections over wide spectral regions are also required for use in modeling studies, like kinetic processes in the presence of high energy radiation. Absolute cross section measurements are however difficult and tiresome, as extreme care has to be devoted to the control of experimental conditions, including density of the sample, geometry of the interaction region, detector efficiency, and the like. So actually rather few data are available for fundamental gaseous targets, notably the noble gases, with a stated accuracy of a few percent. The situation is much more difficult for less volatile or unstable species, many of scientific or technological importance, because of the above mentioned difficulty of precisely characterizing the density of the sample and focal volume.

\footnotetext{
a) Author to whom correspondence should be addressed. Electronic mail: coriani@units.it
}

Experimental total and partial oscillator strength data can be used to validate theoretical concepts and to benchmark computational approximations used to model molecular photo-absorption, photoionization, and ionic photofragmentation processes. While theoretical methods play a fundamental role in providing insight into the experimental information content, the development of more and more sophisticated experimental techniques poses stringent requirements on the quality of the models employed to interpret experimental results and on the accuracy of the underlying chemical-physical descriptions, and prompts the development of more accurate theoretical methodologies.

The theoretical description of the photoionization process is a challenging task in quantum chemistry, as formally complete knowledge of the wave function of the electronic continuum spectrum is required. For this reason, current approaches which include a full treatment of the molecular electronic continuum are well behind pure bound state approaches for the accurate treatment of electron correlation. In this respect, of the many electronic structure methods at hand, coupled cluster (CC) approximations $s^{6,7}$ are considered among the most accurate ones for energies and properties. Nonetheless, their application to the description of molecular photoionization phenomena is somewhat limited, probably because of the difficulty of extracting the asymptotic information common to all correlated methods based on $L^{2}$ finite basis sets.

One elegant and formally convergent way to overcome this problem when computing total photoionization cross sections is to apply the so-called Stieltjes imaging technique to a discretized representation of the continuum part of the spectrum, as pioneered by Langhoff and co-workers. ${ }^{8-10}$ Just a few years ago, Averbukh, Cederbaum, and co-workers proposed to apply the Stieltjes technique to Lanczos pseudo-spectra obtained within the second-order Algebraic Diagrammatic Construction $\mathrm{ADC}(2)$ formalism to describe ionization, ${ }^{11}$ 
autoionization, ${ }^{12}$ and intermolecular decay phenomena. ${ }^{13,14}$ In the spirit of these works, we investigate here the applicability of a similar approach based on the coupled-cluster pseudo-spectra, obtained from a recent formulation of coupled cluster damped linear response theory based on an asymmetric Lanczos algorithm at the coupled cluster single (CCS), coupled cluster singles and iterative approximate doubles (CC2), and coupled cluster single and double (CCSD) levels of theory. ${ }^{15,16}$ Only transitions in the VUV-UV region will be considered in the present study with emphasis on the continuum part of the photo-absorption spectra. ${ }^{17}$

\section{THE COUPLED CLUSTER PSEUDO-SPECTRUM FROM THE ASYMMETRIC LANCZOS PROCEDURE}

The starting point for the computation of the CC pseudo-spectrum, the input quantity for the Stieltjes imaging technique, is the $\mathrm{CC}$ linear response function (diagonal component) for an operator $X^{6}$

$$
\begin{aligned}
\langle\langle X ; X\rangle\rangle_{\omega}= & \sum_{\mu} \eta_{\mu}^{X}\left[t_{\mu}^{X}(\omega)+t_{\mu}^{X}(-\omega)\right] \\
& +\sum_{\mu \nu} \mathrm{F}_{\mu \nu} t_{\mu}^{X}(-\omega) t_{\nu}^{X}(\omega)
\end{aligned}
$$

with the response amplitudes $t_{\mu}^{X}(\omega)$ obtained from the solution of the response equation

$$
(\mathbf{A}-\omega \mathbf{1}) \boldsymbol{t}^{X}(\omega)=-\boldsymbol{\xi}^{X},
$$

where the CC "building-blocks" $\xi_{\mu}^{X}, \eta_{\mu \nu}^{X}, \mathrm{~F}_{\mu \nu}$, and $\mathrm{A}_{\mu \nu}$ are $^{6}$

$$
\begin{gathered}
\xi_{\mu}^{X}=\left\langle\mu\left|e^{-T} X e^{T}\right| H F\right\rangle, \\
\mathrm{A}_{\mu \nu}=\left\langle\mu\left|\exp (-T)\left[H, \tau_{\nu}\right] \exp T\right| H F\right\rangle, \\
\eta_{\mu \nu}^{X}=\left\langle\Lambda\left|\left[X, \tau_{\nu}\right] \exp T\right| H F\right\rangle, \\
\mathrm{F}_{\mu \nu}=\left\langle\Lambda\left|\left[\left[H, \tau_{\mu}\right], \tau_{\nu}\right] \exp T\right| H F\right\rangle .
\end{gathered}
$$

Here, $T$ is the cluster operator, $T=\sum_{\mu} t_{\mu} \tau_{\mu}$, with $t_{\mu}$ indicating the cluster amplitude and $\tau_{\mu}$ the excitation operator at excitation level $\mu$. The symbol $\langle\mu|$ indicates the biorthogonal left manifold of $\tau_{\mu}$, i.e., $\langle\mu|=\langle H F| \tau_{\mu}^{\dagger}$ with $\left\langle H F\left|\tau_{v}^{\dagger} \tau_{\mu}\right| H F\right\rangle=\delta_{v \mu}$, and $\langle\Lambda|=\langle H F|+\sum_{\lambda} \bar{t}_{\lambda}\langle\lambda| e^{-T}$ is known as the coupled cluster Lambda state. We refer to Refs. 6 and 7 for additional information and details on the nomenclature. When the operator $X$ above is one of the three Cartesian components of the electric dipole operator, the linear response function above is equal to minus the corresponding diagonal component of the dipole polarizability tensor, $\alpha_{X X}$.

Within our asymmetric-Lanczos-driven implementation of the coupled-cluster linear response function, ${ }^{15,16}$ the diagonal component of the dipole polarizability can be recast in the diagonal form

$$
\begin{aligned}
\langle\langle X ; X\rangle\rangle_{\omega}^{(k)}= & u^{X} v^{X} \sum_{j} \frac{2 \omega_{j} L_{j 1}^{(k)} R_{1 j}^{(k)}}{\left(\omega-\omega_{j}^{(k)}\right)\left(\omega+\omega_{j}^{(k)}\right)} \\
& -\left(v^{X}\right)^{2} \sum_{j l} \frac{\left[\mathcal{F}_{j l} L_{j 1}^{(k)} L_{l 1}^{(k)}\right]}{\left(\omega-\omega_{j}^{(k)}\right)\left(\omega+\omega_{l}^{(k)}\right)} .
\end{aligned}
$$

Here, $\boldsymbol{L}_{j}^{(k)}$ and $\boldsymbol{R}_{j}^{(k)}$ are left and right eigenvectors, with eigenvalue $\omega_{j}^{(k)}$, obtained diagonalizing the (approximate) tridiagonal representation $\mathbf{T}^{(k)}$ of the CC Jacobian matrix $\mathbf{A}$ generated via the Lanczos algorithm, and $\mathcal{F}_{j l}=\sum_{\mu, v, m, l} \mathrm{~F}_{\mu \nu}$ $Q_{\mu m} Q_{\nu n} R_{m j}^{(k)} R_{n l}^{(k)}$, with $Q_{\mu m}$ indicating the elements of the $\mathbf{Q}$ matrix used to generate $\mathbf{T}^{(k)}$ (see Sec. III below).

The diagonal expression in Eq. (7) is obtained under the assumption that bi-orthogonalized vectors $\eta^{X}$ and $\xi^{X}$ are used as starting vectors in the Lanczos chain procedure outlined in Sec. III,

$$
\begin{gathered}
\mathbf{q}_{1}=\frac{\xi^{X}}{v^{X}}, \quad v^{X}=\left\|\xi^{X}\right\| \\
\mathbf{p}_{1}^{T}=\frac{\eta^{X}}{u^{X}}, \quad u^{X}=\frac{\eta^{X} \xi^{X}}{v^{X}}
\end{gathered}
$$

with the factors $u^{X}$ and $v^{X}$ as norms of such vectors. The superscript $(k)$ is used to indicate that the tridiagonal representation $\mathbf{T}^{(k)}$ is truncated at dimension $k$ (commonly referred to as chain length), which is significantly smaller that the full dimension of the excitation space. Above and in the following, roman summation indices run on the truncated space dimension $k$, whereas greek indices are the conventional CC excitation level indices in the full excitation space.

The oscillator strengths of the individual excitations $(0 \rightarrow j)$ in the Lanczos pseudo-spectrum are computed from the residues of the above linear response function, i.e., for the $X$ Cartesian component,

$$
\begin{aligned}
f_{j}^{X X} & =\frac{2}{3} \omega_{j} \lim _{\omega \rightarrow \omega_{j}}\left(\omega-\omega_{j}\right)\langle\langle X ; X\rangle\rangle_{\omega} \\
& =\frac{2}{3} \omega_{j}^{(k)}\left\{u^{X} v^{X} L_{j 1}^{(k)} R_{1 j}^{(k)}-\left(v^{X}\right)^{2} \sum_{l} \frac{\mathcal{F}_{l j} L_{j 1}^{(k)} L_{l 1}^{(k)}}{\left(\omega_{j}^{(k)}+\omega_{l}^{(k)}\right)}\right\} .
\end{aligned}
$$

Increasing the chain length value, an increasing amount of pseudo-eigenvectors will converge to true accurate eigenvectors, allowing standard $\mathrm{CC}$ calculations of transition properties.

The CCS and CCSD members of the coupled cluster hierarchy employed in this work are defined by truncations in the cluster operator to include the complete manifolds of single (CCS) and single and double excitations (CCSD), respectively. The CC2 method is derived from CCSD by introducing certain additional approximations in the manifolds of double excitations, ${ }^{19}$ which yields reduced computational scaling compared to CCSD. 


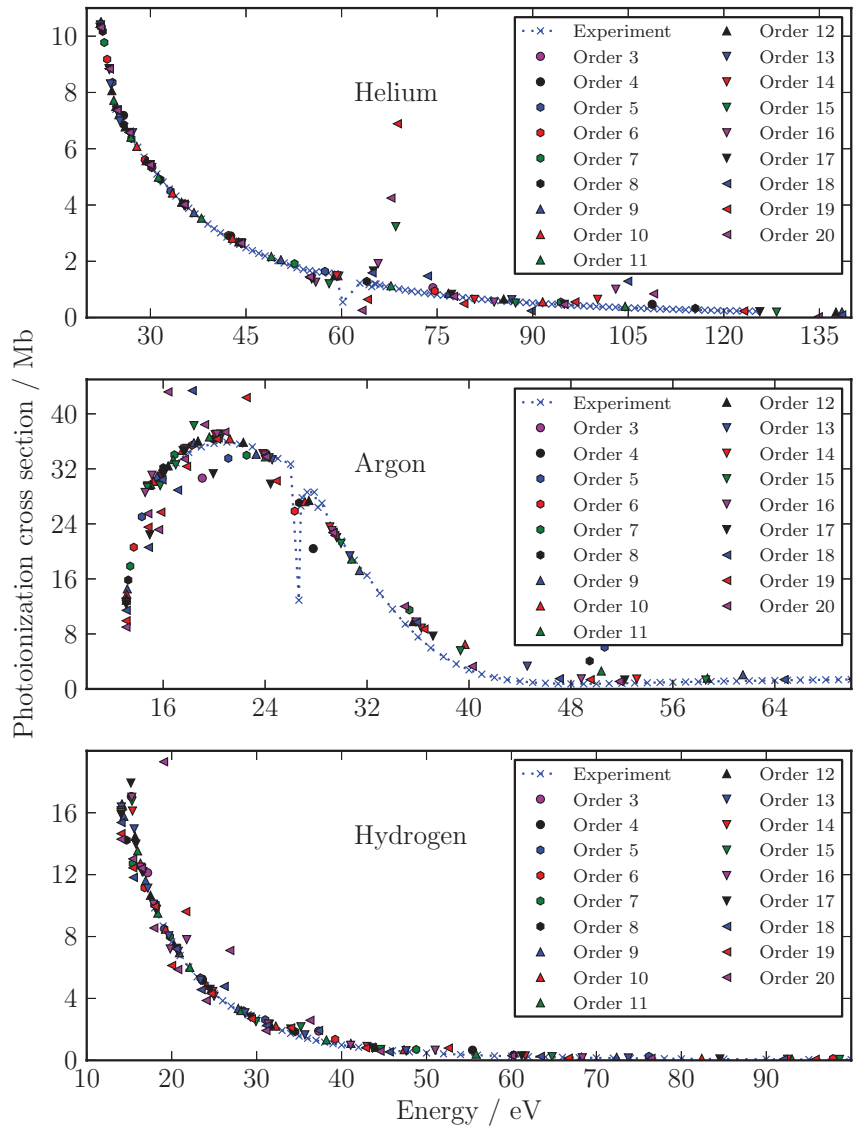

FIG. 1. The full set of CCSD results obtained for helium (upper panel), argon (middle panel), and $\mathrm{H}_{2}$ (lower panel) comprising all computed Stieltjes orders from $l=3$ to 20 . The blue saltires are experimental points.

\section{THE ASYMMETRIC LANCZOS ALGORITHM}

The Lanczos algorithm is essentially a method to generate a (smaller) tridiagonal matrix $\mathbf{T}$ from a generic matrix $\mathbf{A} .{ }^{18}$ In its asymmetric variant, required when the matrix $\mathbf{A}$ is not Hermitian, as it is the case for our coupled cluster Jacobian, the tridiagonal matrix is computed as

$$
\begin{gathered}
\mathbf{T}=\mathbf{P}^{T} \mathbf{A} \mathbf{Q}=\left(\begin{array}{ccccc}
\alpha_{1} & \gamma_{1} & 0 & \cdots & 0 \\
\beta_{1} & \alpha_{2} & \gamma_{2} & 0 & \vdots \\
0 & \beta_{2} & \alpha_{3} & \ddots & 0 \\
\vdots & 0 & \ddots & \ddots & \gamma_{k-1} \\
0 & \cdots & 0 & \beta_{k-1} & \alpha_{k}
\end{array}\right), \\
\mathbf{T}=\mathbf{P}^{T} \mathbf{A Q}, \quad \mathbf{P}^{T} \mathbf{A}=\mathbf{T P}^{T}, \quad \mathbf{A Q}=\mathbf{Q T},
\end{gathered}
$$

with

$$
\mathbf{P}^{T} \mathbf{Q}=\mathbf{1}
$$

by means of an iterative procedure. The matrices $\mathbf{P}^{T}$ and $\mathbf{Q}$ collect the trial vector pairs $\mathbf{p}_{i}^{T}$ and $\mathbf{q}_{i}$ in, respectively, their rows and columns. The iterative procedure is initiated by selecting a couple of start vectors, $\mathbf{q}_{1}$ and $\mathbf{p}_{1}^{T}$, and computing $\alpha_{1}=\mathbf{p}_{1}^{T} \mathbf{A} \mathbf{q}_{1}$. As already mentioned in Sec. II, for the spe-

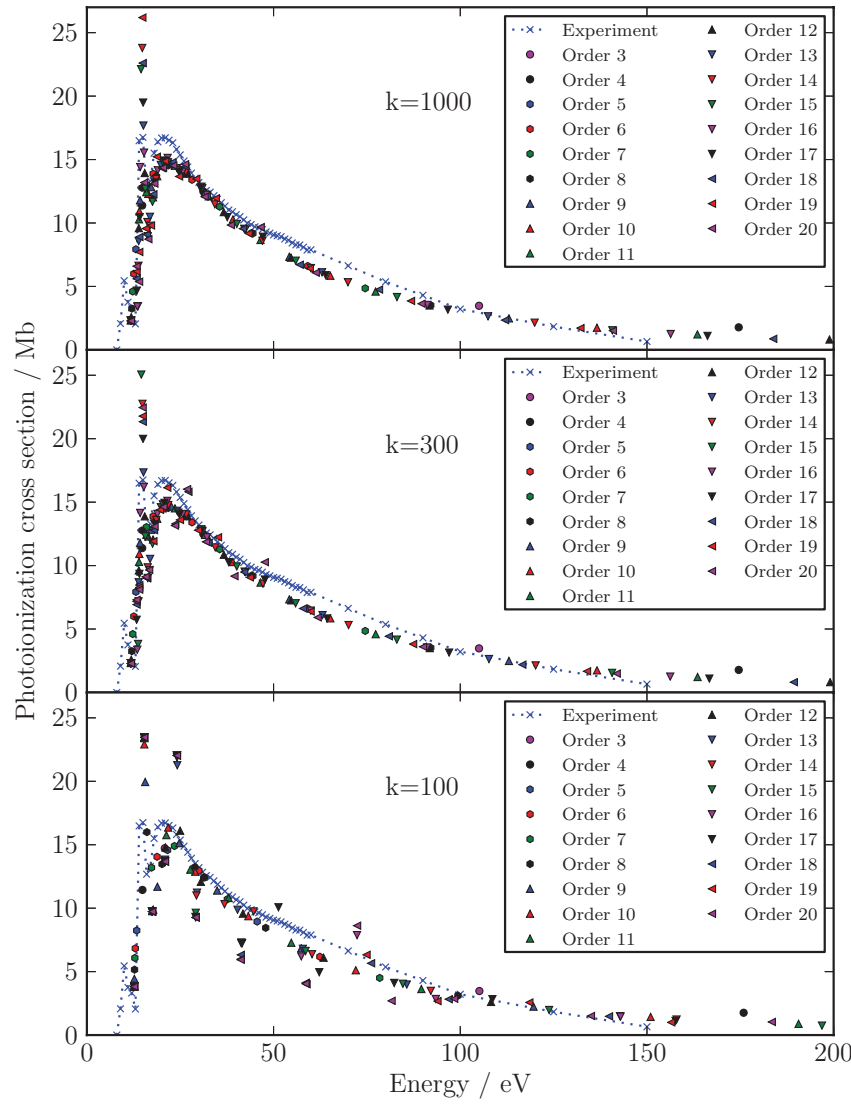

FIG. 2. HF. Dependence of the computed CCSD cross-section profile on the chain length $k$. The connected blue saltires are experimental points from Ref. 32 .

cific case considered here it proves advantageous to use the biorthogonalized vectors $\eta^{X}$ and $\xi^{X}$ as starting seeds, since this yields a diagonal representation of the linear response function and of the oscillator strengths.

The trial bases (hence the matrices $\mathbf{P}^{T}, \mathbf{Q}$, and $\mathbf{T}$ ) are progressively enlarged generating first the vectors

$$
\mathbf{r}_{i}=\left(\mathbf{A} \mathbf{q}_{i}-\gamma_{i-1} \mathbf{q}_{i-1}-\alpha_{i} \mathbf{q}_{i}\right)
$$

$$
\mathbf{s}_{i}^{T}=\left(\mathbf{p}_{i}^{T} \mathbf{A}-\beta_{i-1} \mathbf{p}_{i-1}^{T}-\alpha_{i} \mathbf{p}_{i}^{T}\right),
$$

which in turn determine the elements $\beta_{i}$ and $\gamma_{i}$ in force of the condition

$$
\beta_{i} \gamma_{i}=\mathbf{s}_{i}^{T} \mathbf{r}_{i}
$$

that is, for instance,

$$
\begin{gathered}
\beta_{i}=\sqrt{\mathbf{s}_{i}^{T} \mathbf{r}_{i},} \\
\gamma_{i}=\left(\mathbf{s}_{i}^{T} \mathbf{r}_{i}\right) \beta_{i}^{-1},
\end{gathered}
$$

and the new trial basis vectors

$$
\begin{gathered}
\mathbf{p}_{i+1}^{T}=\gamma_{i}^{-1} \mathbf{s}_{i}^{T}, \\
\mathbf{q}_{i+1}=\beta_{i}^{-1} \mathbf{r}_{i} .
\end{gathered}
$$

Note that $\beta_{0}=0$ in the first iteration. Furthermore, the new basis vectors $\mathbf{p}_{i+1}^{T}$ and $\mathbf{q}_{i+1}^{T}$ are usually explicitly 


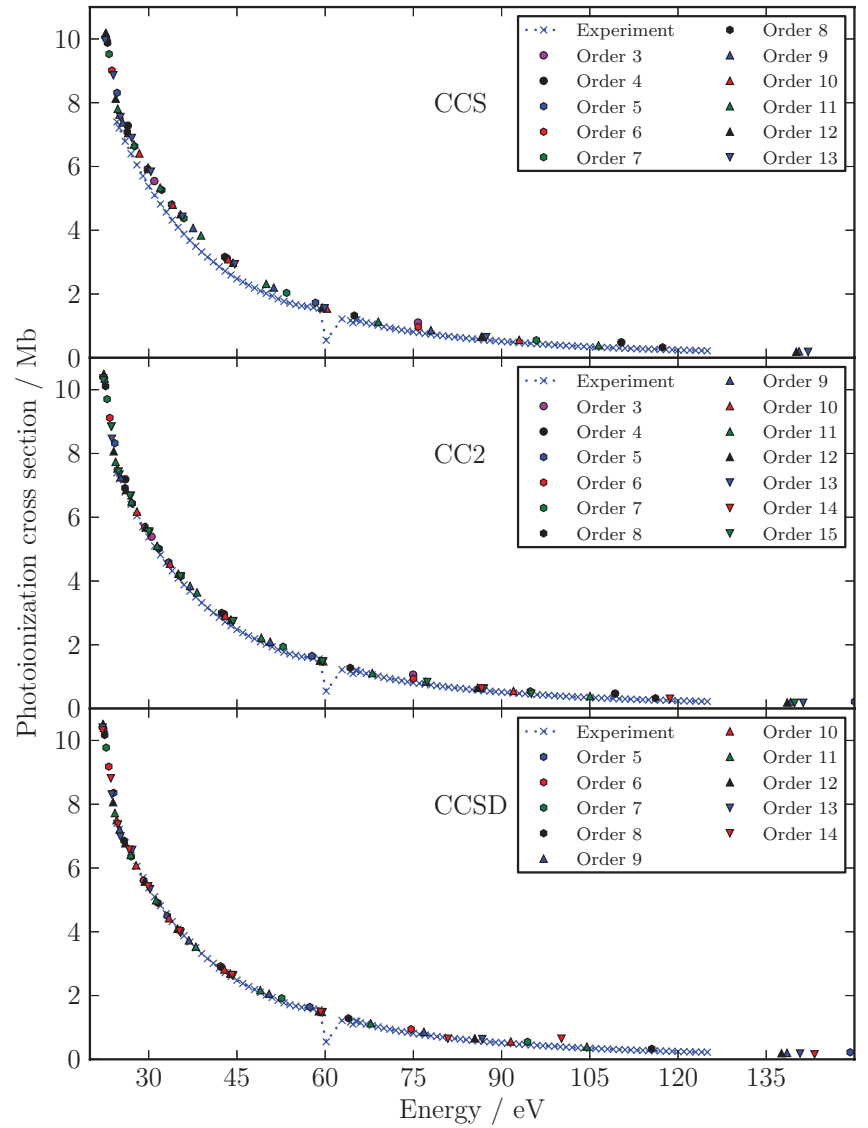

FIG. 3. Helium. CCS (upper panel), CC2 (middle panel), and CCSD (lower panel) results obtained using the aug-cc-pV5Z set supplemented with $(10 s 10 p 10 d)$ continuum-like functions. For CCS, the chain length $k$ is equal to the full single-excitation-space dimension in the given basis set; for $\mathrm{CC} 2$ and CCSD, the chain length $k$ is equal to the full excitation space dimension in the given basis set. Note that CCSD $=$ FCI. Experimental points (connected blue saltires) are from Ref. 30. Stieltjes orders $l$ are given in the legend.

biorthogonalized ( $\mathbf{p}_{i}^{T} \mathbf{q}_{j}=\delta_{i j}$ ) to the set of all previous basis vectors to avoid (near-)linear dependencies. Also note that the procedure outlined here is the ordinary form of the (asymmetric) Lanczos algorithm as implemented in our code, ${ }^{15,16}$ but that it could be further generalized into its block or band variants. $^{20-22}$

\section{THE STIELTJES IMAGING PROCEDURE}

In a finite basis set calculation, a set of discrete excitation energies and oscillator strengths is obtained

$$
\left\{\omega_{i}, f_{i}\right\}_{i=1, k}
$$

which actually represent - above the ionization threshold wavepackets in the true continuum that are very basis-set dependent and do not possess well defined convergence properties. It is possible, however, to converge the spectral moments of the Hamiltonian

$$
S(-m)=\sum_{i=1}^{k} \omega_{i}^{-m} f_{i}, \quad m=0, \ldots, 2 n-1,
$$

where $n \ll k$. From these, it is possible to extract convergent principal pseudo-spectra $\left(\omega_{j}^{p}, f_{j}^{p}\right)_{j=1, \ldots, l}$ which rep-

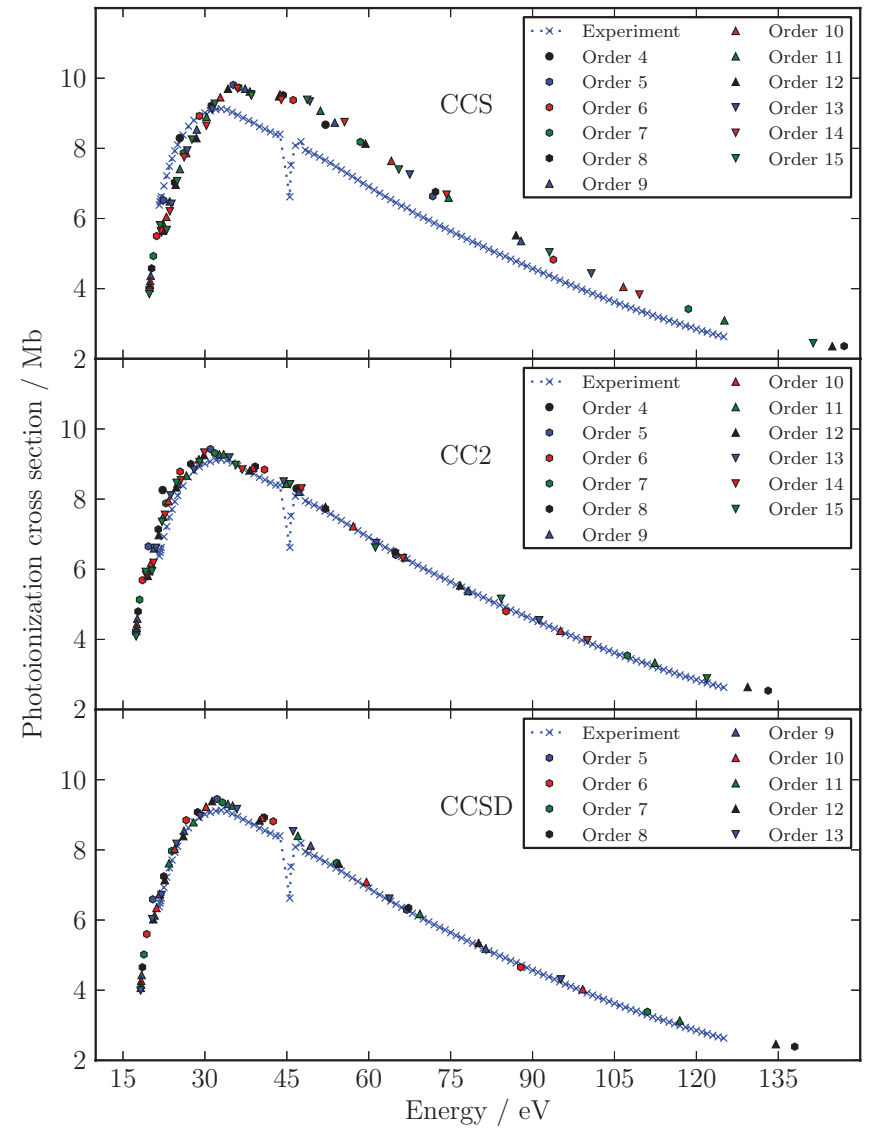

FIG. 4. Neon. CCS (upper panel), CC2 (middle panel), and CCSD (lower panel) results obtained using the aug-cc-pCVQZ basis plus (10s10p10d) continuum-like functions. For CCS, the chain length $k$ is equal to the full single-excitation space dimension in the given basis set; for CC2 and CCSD, $k=1500$. Experimental points (connected blue saltires) are taken from Ref. 30.

resent the oscillator strength distribution at the quadrature points $\omega_{j}^{p}$. $^{23}$ Given $2 n$ spectral moments, up to $n-1$ principal pseudo-spectra can be obtained, which, with increasing $n$, yield an ever denser representation of the continuous distribution. Each principal pseudo-spectrum consists of $l$ pairs $\left(\omega_{j}^{p}, f_{j}^{p}\right)_{j=1, \ldots, l}$, with the number of elements $l$ varying from 2 to $n$. We refer to $l$ as Stieltjes order of the principal pseudospectrum. Different algorithms are used to generate the $n-$ 1 principal pseudo-spectra. ${ }^{23,24}$ Here, we employ a method proposed by Langhoff et al. ${ }^{8,10}$

The Stieltjes derivatives

$$
\tilde{g}\left(\omega_{j}\right)=\frac{1}{2} \frac{f_{j+1}^{p}+f_{j}^{p}}{\omega_{j+1}^{p}-\omega_{j}^{p}}, \quad j=1, \ldots, l-1,
$$

representing discrete points in the continuum oscillator strength distribution at frequencies $\omega_{j}=\frac{1}{2}\left(\omega_{j+1}^{p}+\omega_{j}^{p}\right)$, are then computed from the principal pseudo-spectrum pairs of each order. From the Stieltjes derivatives $\tilde{g}\left(\omega_{j}\right)$, point-wise values of the absorption cross section $\sigma(\omega)$ at $\omega=\omega_{j}$ are finally obtained according to

$$
\sigma\left(\omega_{j}\right)=\frac{2 \pi^{2}}{c} \tilde{g}\left(\omega_{j}\right)
$$

Of course only the converged spectral moments have to be employed and, in practice, with affordable bases, a rather 


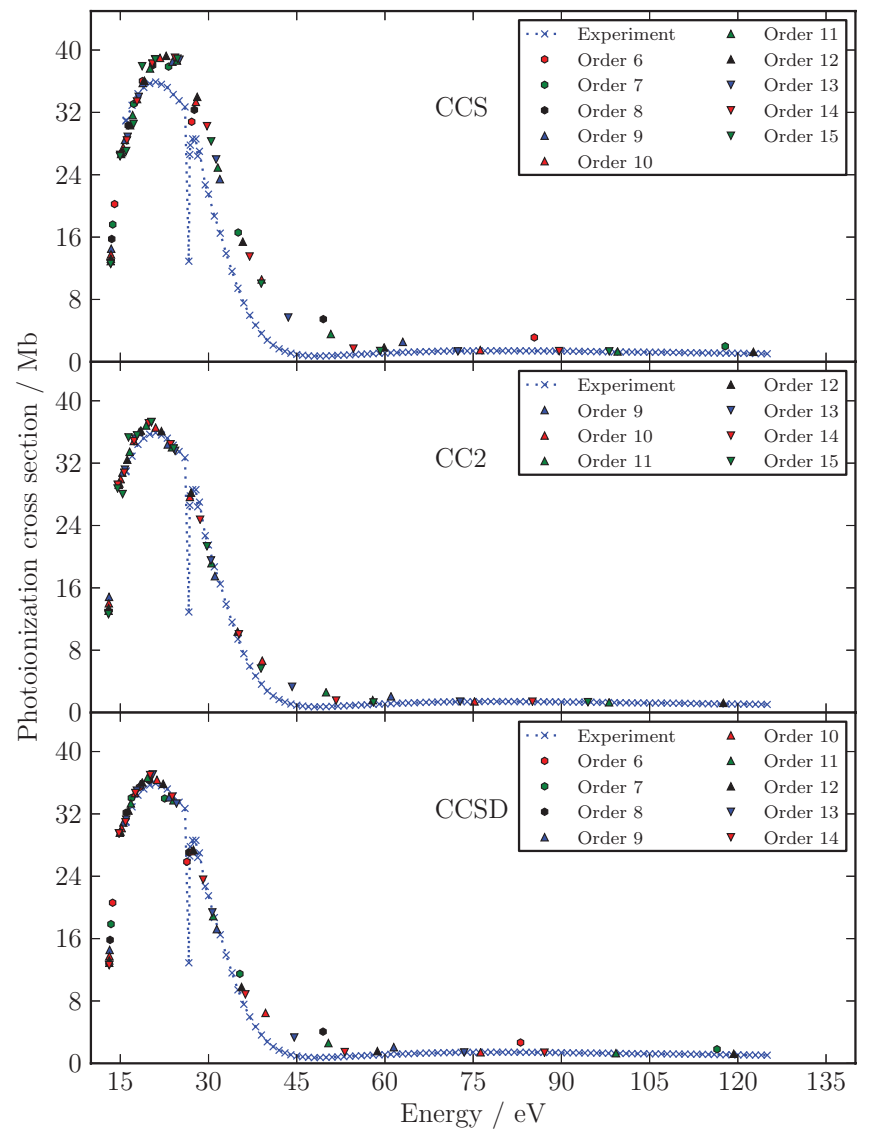

FIG. 5. Argon. CCS (upper panel), CC2 (middle panel), and CCSD (lower panel) cross-sections obtained using the aug-cc-pCV5Z basis set supplemented with $(10 s 10 p 10 d)$ continuum-like functions. For CCS, the chain length $k$ is equal to the dimension of the single-excitation space, whereas $k=1200$ for CC 2 and CCSD. Experimental points (connected blue saltires) are taken from Ref. 30.

limited number can be reached, after which scattered values begin to appear, a reflection of the underlying pseudospectrum generated by the finite basis. Since many cross sections are weakly structured, that does not pose a serious problem, although it tends to broaden sharper features which may appear in the spectra.

To complete this section, it is appropriate to mention that the even coupled-cluster spectral (also known as Cauchy) moments $S(-2 m)$ can also be obtained by the analytic procedure of Ref. 25, which computes the dispersion coefficients of the dipole polarizability - and hence the Cauchy moments - as frequency derivatives of the coupled cluster linear response function. This is however not sufficient in our context, as both odd and even Cauchy moments are required for the Stieltjes imaging. In the present study, we nonetheless used the even CC Cauchy moments computed according to Ref. 25 to check the convergence of the even moments generated by the Lanczos pseudo-spectrum.

\section{COMPUTATIONAL DETAILS}

Calculations of the CC pseudo-spectra have been performed at the CCS, CC2, and CCSD levels using the asymmetric Lanczos algorithm we have recently implemented ${ }^{15,16}$

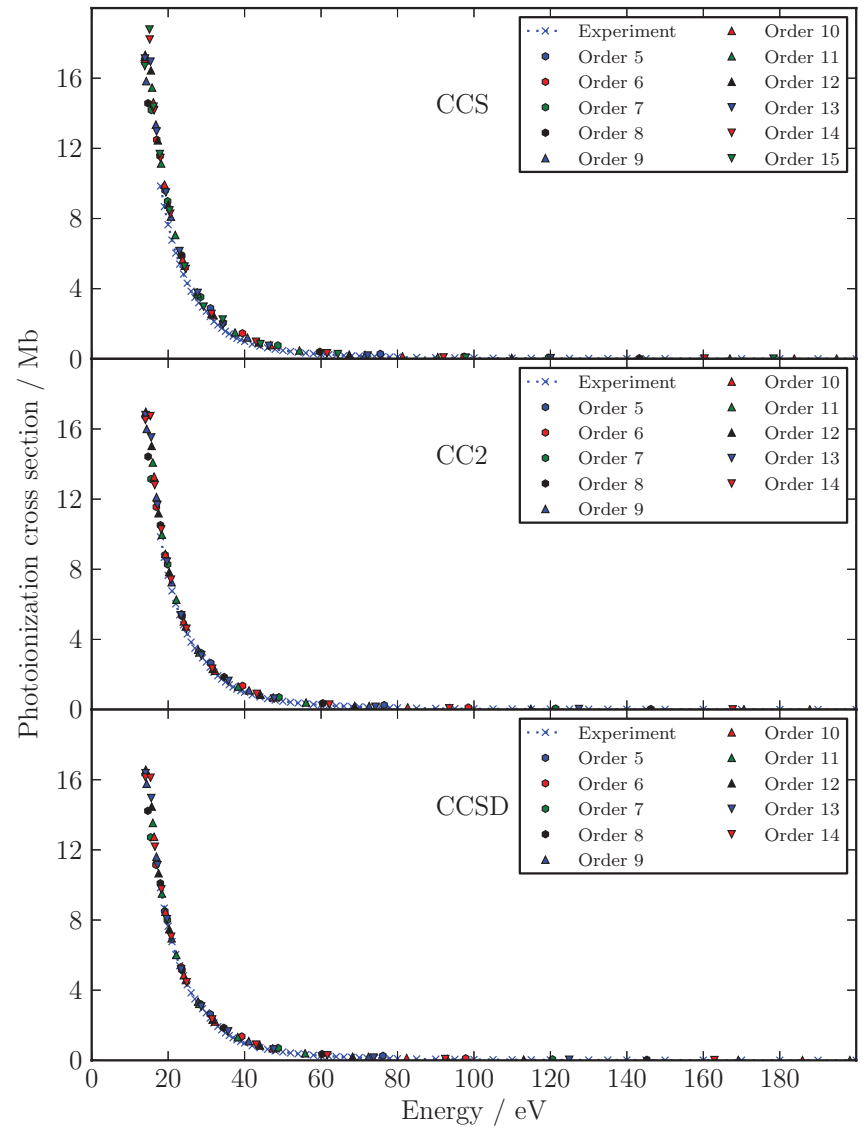

FIG. 6. $\mathrm{H}_{2}$. CCS (upper panel), CC2 (middle panel), and CCSD (lower panel) cross-sections obtained using the aug-cc-pVQZ set + (10s10p10d) continuum-like functions. The chain length $k$ is equal to the full-excitation space dimension in the given basis set. Experimental data (connected blue saltires) are from Ref. 31.

in the Dalton program package, ${ }^{26}$ which has been interfaced to our own Stieltjes imaging procedure.

For all systems, correlation consistent basis sets of Dunning and co-workers have been employed, specifically singly (aug-) and doubly (d-aug-) augmented valence and corevalence sets cc-p $(\mathrm{C}) \mathrm{VXZ}$ with $\mathrm{X}=\mathrm{T}, \mathrm{Q}, 5$, depending on the system. As the Stieltjes procedure is extremely sensitive to the description of the continuum, additional continuum-like Gaussian basis functions - generated using the expression (cf. Eq. (20)) and parameters given by Kaufmann and co-workers in Ref. 27 - were added in the center of mass of all investigated systems, with quantum number $n$ ranging from 1 to 8 , 9 , or 10 depending on the specific case at hand.

Experimental equilibrium geometries were used for all molecular species, taken from the compilation in Ref. 28. All electrons were correlated in all calculations, unless otherwise specified.

\section{DISCUSSION OF RESULTS}

We start this section with a discussion of how the crosssection points resulting from the Stieltjes procedure shown in the following Figs. 1 to 11 were selected. The Stieltjes imaging procedure is known to be numerically unstable ${ }^{24,29}$ and its results to degrade quickly when going to higher 


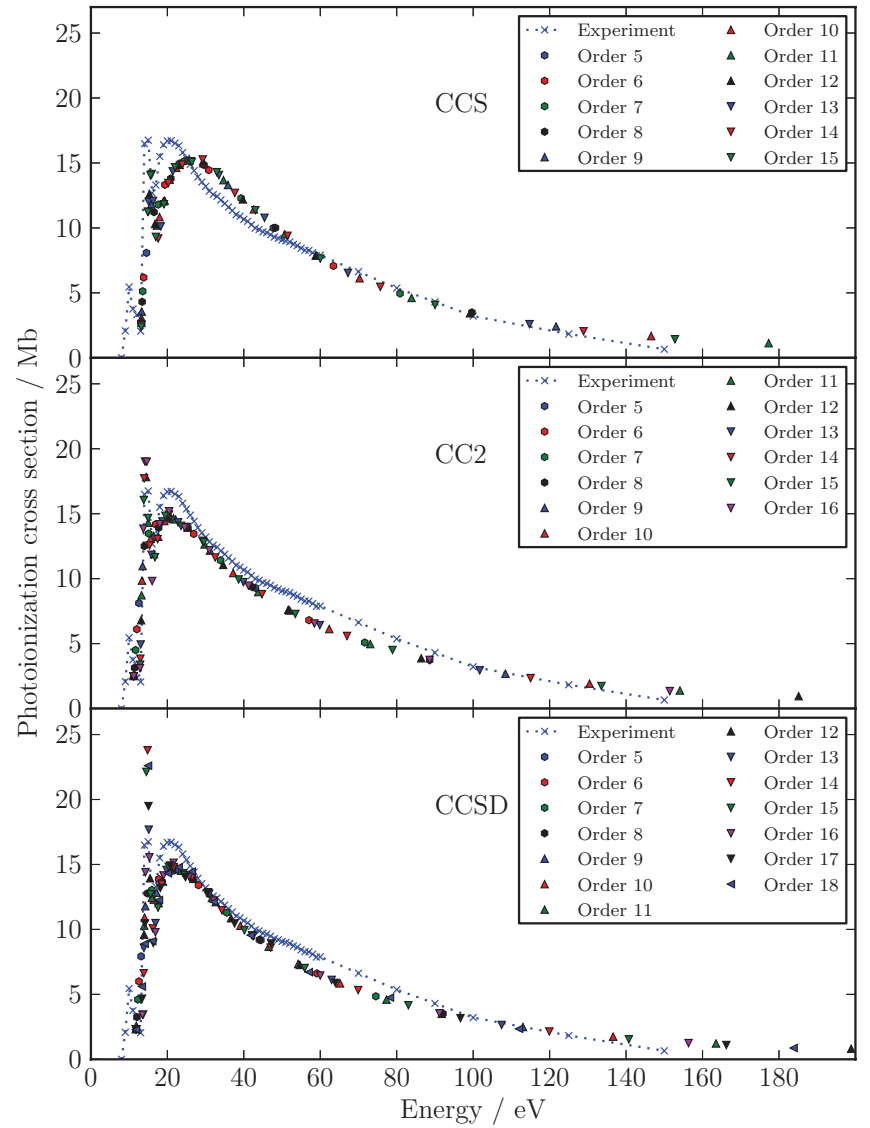

FIG. 7. HF. CCS (upper panel), CC2 (middle panel), and CCSD (lower panel) cross-sections obtained with basis set d-aug-pCVTZ + $(10 s 10 p 10 d)$ continuum-like functions. For CCS, the chain length $k$ is equal to the dimension of the single-excitation space, whereas $k=2000$ for CC2 and CCSD. Experimental data (connected blue saltires) are from Ref. 32 .

spectral moments (and therefore higher Stieltjes orders). If the spectral moments are not well converged, the points obtained from the Stieltjes procedure appear away from the true results and are usually scattered. This happens mostly for the lowest and highest Stieltjes orders. According to the literature, ${ }^{11,29}$ it is therefore common practice to use Stieltjes orders from 6 to 12 as converged and relevant, and to discard the points obtained for all other "unstable" orders. As an illustration of this, in Fig. 1 we present the full set (i.e., no points removed) of results of the Stieltjes imaging applied to the Lanczos spectra of $\mathrm{He}, \mathrm{Ar}$, and $\mathrm{H}_{2}$. For instance, in the case of helium, the points obtained for Stieltjes orders $l \geq 14$ appear off the main curve. For argon, both the lower and higher order points are scattered. Remarkably, the results seem to be slightly more stable for the studied molecules than for the atoms, see, e.g., $\mathrm{H}_{2}$ in Fig. 1.

For all system investigated, we analyzed the points generated by each Stieltjes order and, if necessary, removed the orders which by visual inspection appeared not to be converged. Only these "filtered" results are displayed in the remaining Figs. 2 to 11. The range of converged Stieltjes orders is different in each case and varies between 3 and 20; nonetheless, for all of the systems, the orders in-between 6 and 12 always yield good results. Note that we are primarily concerned with the continuum profiles above the ionization thresholds, even though we obtain points below that range.

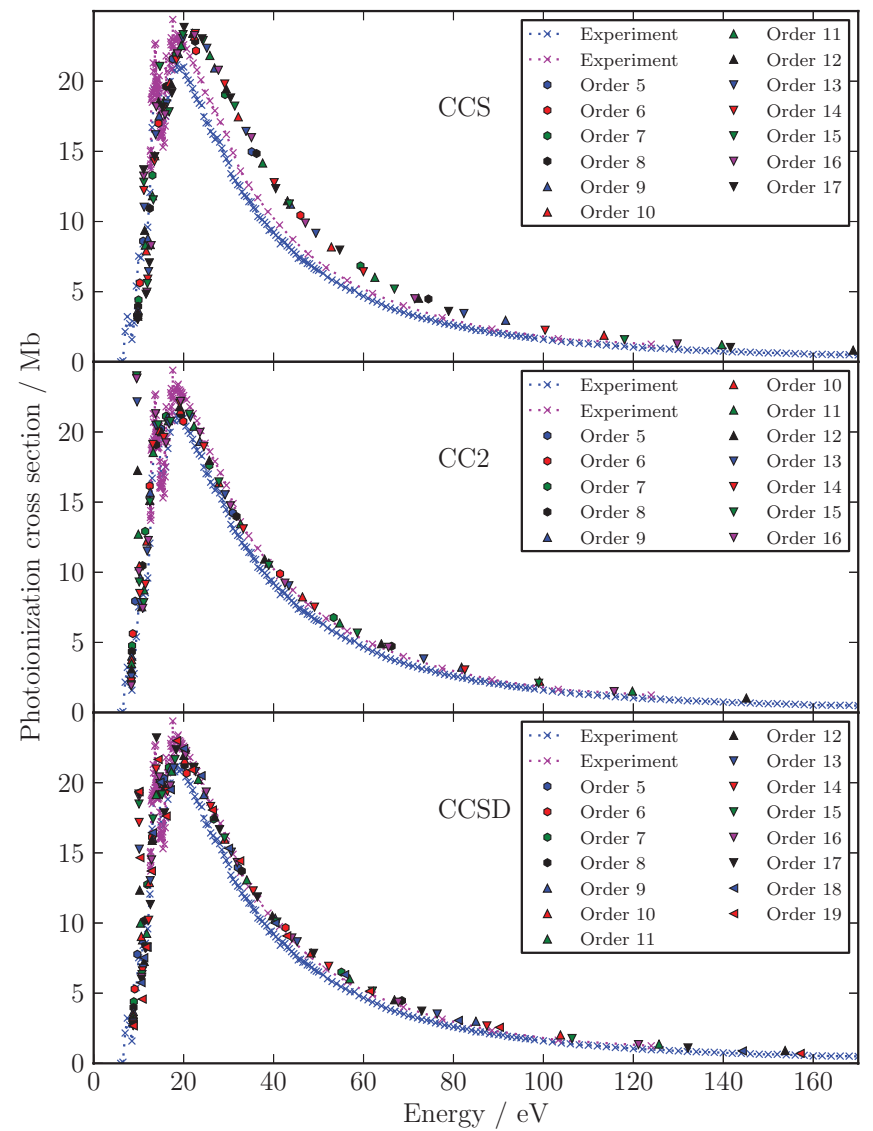

FIG. 8. $\mathrm{H}_{2} \mathrm{O}$. CCS (upper panel), CC2 (middle panel), and CCSD (lower panel) cross-sections obtained with basis set aug-pCVTZ $+(10 s 10 p 10 d)$ continuum-like functions. The Stieltjes orders adopted are given in the legend. For CCS, the chain length $k$ is equal to the dimension of the singleexcitation space, whereas $k=1500$ for CC2 and CCSD. Experimental data are from Ref. 33 (connected blue saltires) and from Ref. 34 (connected pink saltires).

Another aspect that may play a critical role in the assessment of the quality of the computed cross section is the choice of the dimension of the chain length $k$. In general, we found that relative small chain lengths were sufficient to obtain well converged cross-section profiles. This behaviour is clearly illustrated in Fig. 2, where the Stieltjes points obtained, at the CCSD level, for the hydrogen fluoride molecule with three different chain lengths, $k=100$ (lower panel), $k=300$ (middle panel), and $k=1000$ (upper panel), are given. As shown, apart from the lower value, the profiles for the larger chain lengths are practically indistinguishable. The same rapid convergence is observed if one considers the difference between the even spectral moments yielded by our (truncated) Lanczos chain approach and those obtained from the analytic calculation of the dispersion coefficients according to Ref. 25. The lowest orders are basically identical for $k=300$; the highest (and numerically very large) moments have of course rather large absolute deviations, but in relative terms the two values differ by at most $0.07 \%$. This rapid convergence is in our opinion consistent with the fact that we are only addressing the region of valence photoionization, which is expected to be dominated by the fast convergence of the individual states at the lower (and upper) edge of the eigenvalue spectrum (and 


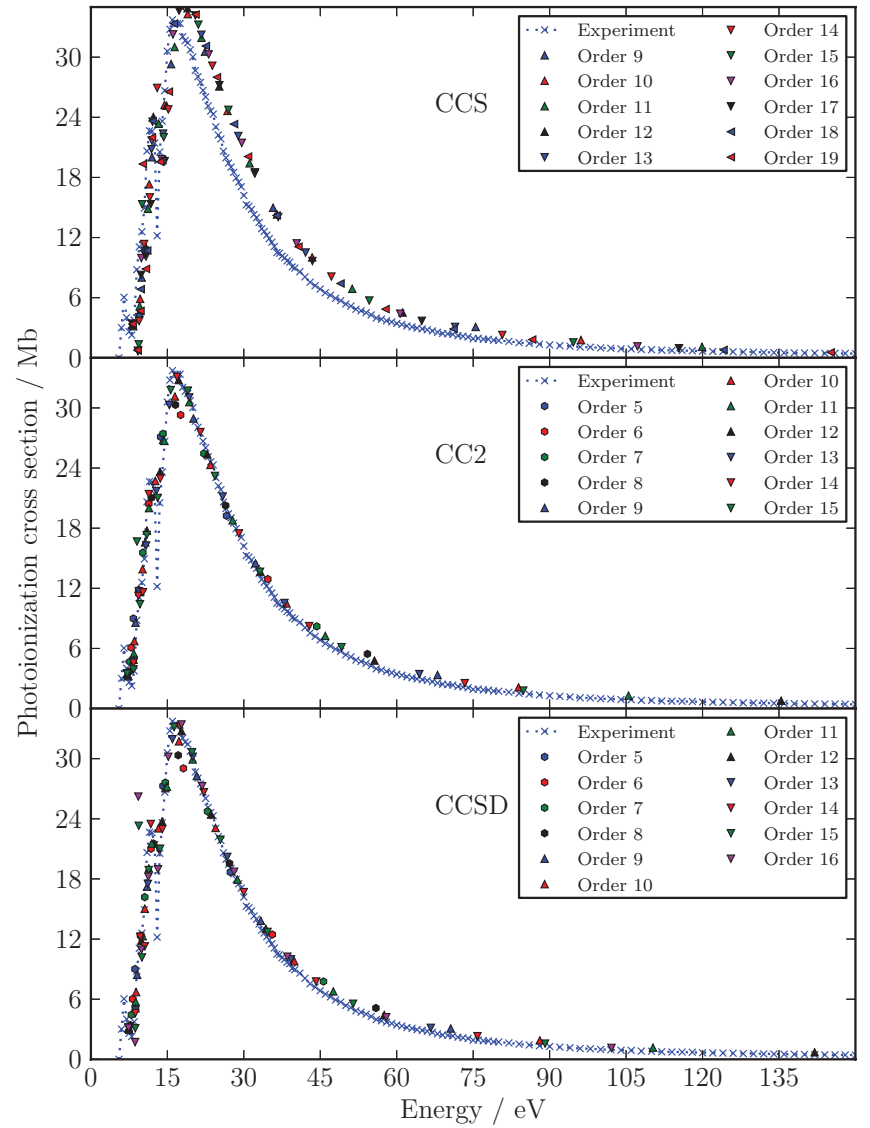

FIG. 9. $\mathrm{NH}_{3}$. CCS (upper panel), $\mathrm{CC} 2$ (middle panel), and CCSD (lower panel) cross-sections obtained with basis aug-cc-pCVTZ+ $(9 s 9 p 9 d)$ continuum-like functions. The Stieltjes orders adopted are given in the legend. For CCS, the chain length $k$ is equal to the dimension of the singleexcitation space, whereas $k=1800$ for CC2 and CCSD. Experimental data (connected blue saltires) are from Ref. 35 .

of the dipole polarizability obtained thereof), a well known characteristic of the Lanczos approach. In any case, in order to minimize the influence of the chain length dimension on the computed profiles, we have in all cases adopted chain length dimensions of the order of 1000-2000, depending on the system. For the two-electron systems $\mathrm{He}$ and $\mathrm{H}_{2}$ the full excitation dimension space was always used.

The most crucial point in the assessment of the accuracy of the Stieltjes imaging procedure in general, and coupled to the CC methods in particular, is the choice of basis set. Naively, one could imagine that adopting a very large basis set would be sufficient to obtain good pseudo-spectra and therefore spectral moments on which the Stieltjes imaging can be safely performed to yield good cross-section profiles. This is however not quite the case. The chosen basis set must in all cases contain some of the physical characteristics of the continuum wave function, along with polarization and diffuse functions for a reasonable description of the region of discrete excitations. We found that inclusion of sets of "continuumlike" Gaussian basis functions of Kaufmann et al. ${ }^{27}$ - with the optimal exponents given in Eq. 20 of Ref. 27, not to be confused with the Gaussian basis functions for Rydberg states in Eq. (18) of the same paper - was a sine-qua-non condition to generate sufficiently accurate spectral moments on which

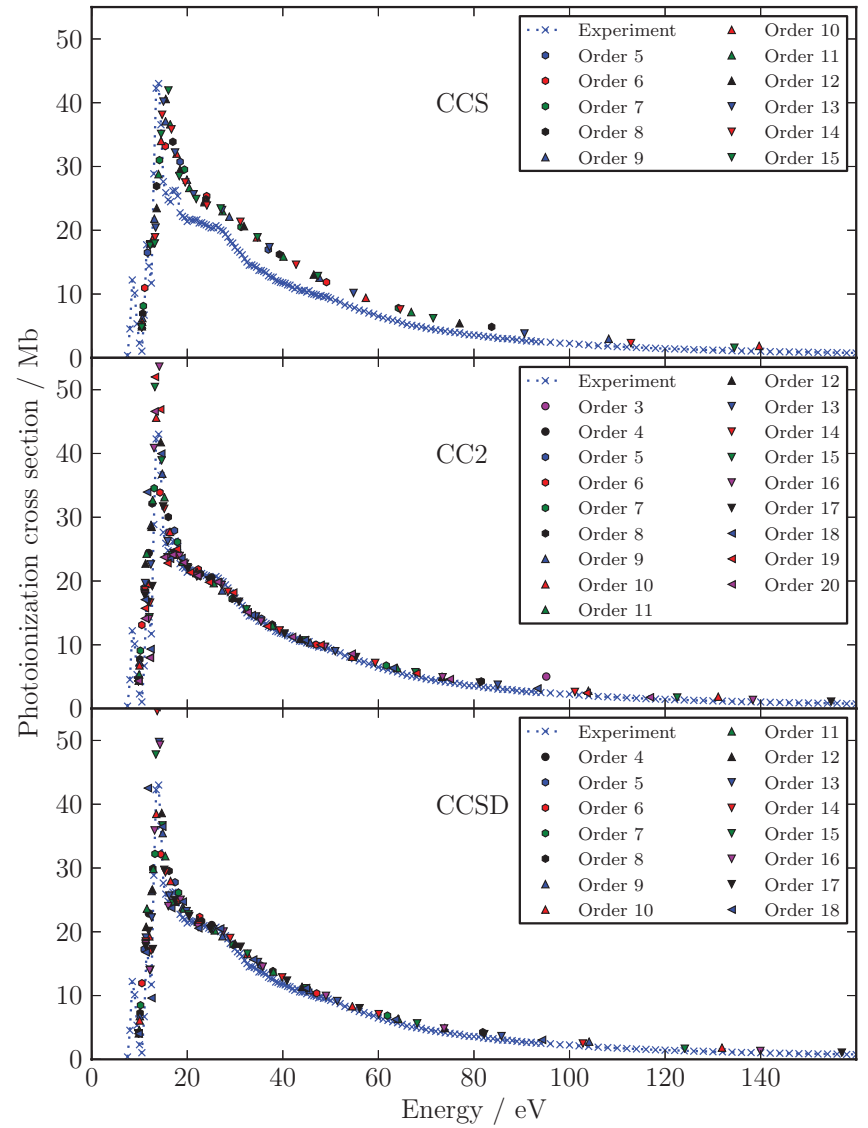

FIG. 10. CO. CCS (upper panel), CC2 (middle panel), and CCSD (lower panel) cross-sections obtained with basis set aug-cc-pCVTZ+(10s10p10d) continuum-like functions. The Stieltjes orders adopted are given in the legend. For CCS, the chain length $k$ is equal to the dimension of the singleexcitation space, whereas $k=1500$ for CC2 and CCSD. Experimental data (connected blue saltires) are from Ref. 36.

the Stieltjes procedure could be applied with confidence. A particularly challenging case in terms of basis set selection proved to be the carbon dioxide molecule, for which good agreement with the experimental profiles in the region between 20 and $30 \mathrm{eV}$ could only be attained upon inclusion of a second shell of diffuse functions, whereas with only one augmentation shell unphysical features tended to emerge in this region.

To conclude this section, we turn now to the final comparison of our results with the experimental cross-section profiles. The photoionization cross-sections of all atomic and molecular systems considered here have been the subject of several investigations in the past, both experimentally and theoretically, and we refer to the book by Berkowitz ${ }^{17}$ for a compilation of results up to 2002. For the noble gases $\mathrm{He}, \mathrm{Ne}$, and $\mathrm{Ar}$, the most recent experimental reinvestigation is probably the one by Samson and Stolte, ${ }^{30}$ and we chose this as main reference for the comparison with our results in Figures $3-5$. Note that both $\mathrm{He}$ and $\mathrm{Ne}$ were also considered in the recent computational ADC(2) study of Gokhberg et al. ${ }^{11}$ For the molecules, our key experimental references are the measurements by Samson and Haddad ${ }^{31}$ for $\mathrm{H}_{2}$, by Carnovale et al. ${ }^{32}$ for hydrogen fluoride, by Haddad and Samson $^{33}$ as well as by Chan et al. ${ }^{34}$ for water, by Burton et al. ${ }^{35}$ for ammonia, by 


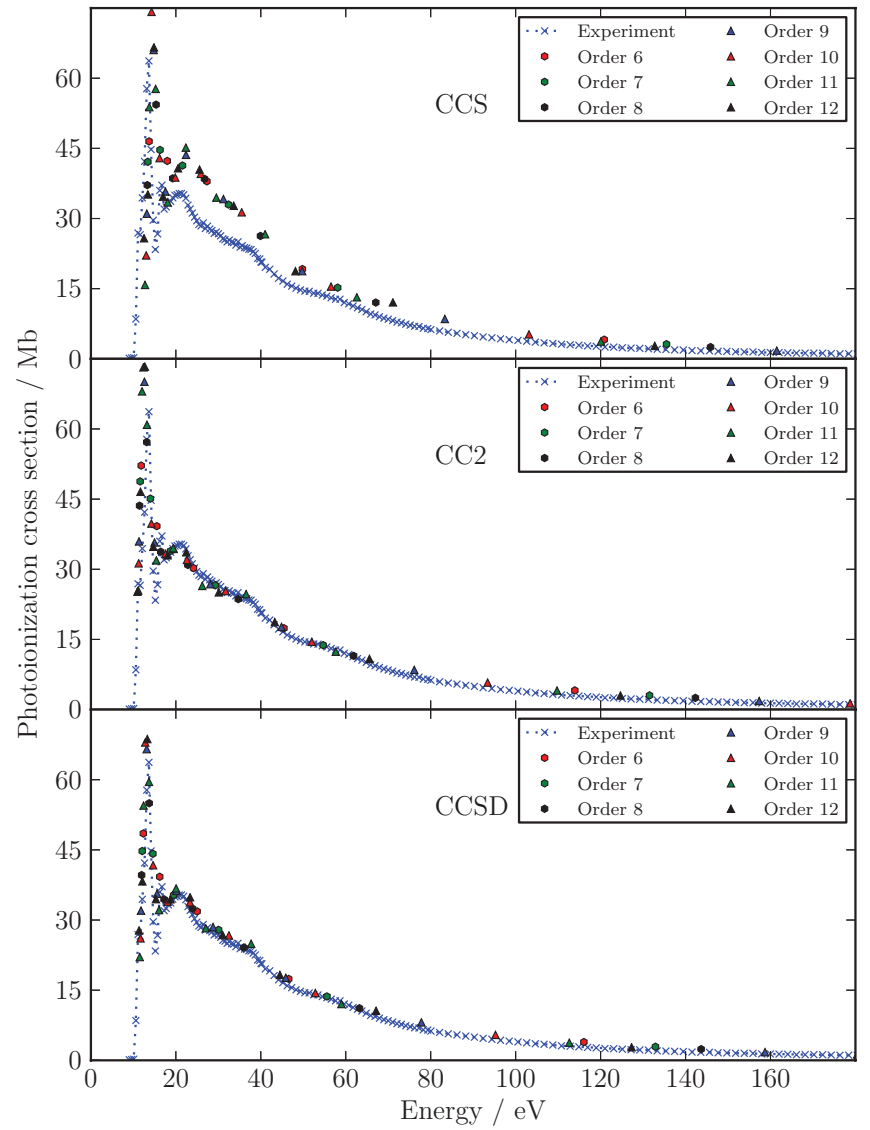

FIG. 11. $\mathrm{CO}_{2}$. CCS (upper panel), $\mathrm{CC} 2$ (middle panel), and CCSD (lower panel) cross-sections obtained with basis set d-aug-cc-pVTZ+ $(8 s 8 p 8 d)$ continuum-like functions. The core electrons were kept frozen. For CCS, the chain length $k$ is equal to the dimension of the single-excitation space, whereas $k=1000$ for CC2 and CCSD. Experimental data (connected blue saltires) are from Ref. 37.

Chan et al. ${ }^{36}$ for carbon monoxide, and by Chan et al. ${ }^{37}$ for carbon dioxide.

For each species, the cross-section points generated from the Stieltjes imaging procedure at each level of the CC hierarchy are compared vis-a-vis with the experimental data points (connected blue saltires), see Figs. 3-11. In the upper panel of each figure, the results for the CCS method using a chain length that corresponds to the full space of single excitations are reported; in the middle and lower panels the results for, respectively, CC2 and CCSD using the value of the chain length specified in the figure caption (same value for both methods) are shown, together with the above mentioned experimental data points.

For all treated systems, the CCS profiles always overshoot the experimental ones. The CC2 and CCSD methods are found, visually at least, of comparable quality, and yield in general cross-section points that are in rather good agreement with the experimental profiles. The most pronounced deviations from the experimental profiles are observed for hydrogen fluoride, see Fig. 7, where the computed cross-section points (for CC2 and CCSD) are systematically below the experimental ones. However, in view of the observed agreement for all other species, we suspect that a rescaling of the (rather old) experimental results may be required.

\section{CONCLUSIONS}

We have presented pilot results of a computational procedure that couples CC Lanczos pseudo-spectra to Stieltjes imaging to simulate the photo-absorption cross section spectra in the region of the excitations from valence orbitals to the continuum for a series of atoms and molecules. CCS is in general found to overestimate the photoionization profiles, and in many instances it is quite similar to previous staticexchange CI results ${ }^{38,39}$ indicating that interchannel coupling included in CCS is of minor importance for total cross section. On the contrary, both CC2 and CCSD yield in general quite similar cross-section profiles, which are in rather good agreement with the corresponding experimental data, showing the importance of dynamical correlation contribution for a quantitative reproduction of total cross section values. The methodology appears therefore to be quite promising and it is being tested further on larger molecular systems. In perspective, particularly interesting is the good performance of the $\mathrm{CC} 2$ approximation, in view of the fact that, with the necessary technical developments in place, it may give access to the possibility to treat large molecular systems. ${ }^{40,41}$

\section{ACKNOWLEDGMENTS}

Computer time from Danish Center for Scientific Computing is acknowledged. This work has been supported by the Italian PRIN2009 funding scheme and by the FP7-PEOPLE2009-IEF funding scheme (S.C., Project No. 254326). The COST-CMTS Action CM1002 "COnvergent Distributed Environment for Computational Spectroscopy (CoDECS)" is also acknowledged.

${ }^{1}$ The Opacity Project Team, in The Opacity Project Vol. 1 (Institute of Physics Publishing, Bristol, UK, 1995), pp. 1-29.

${ }^{2}$ M. J. Seaton, Mon. Not. R. Astron. Soc. 362, L1 (2005).

${ }^{3}$ J. S. Hirsch, E. T. Kennedy, A. Neogi, J. T. Costello, P. Nocolosi, and L. Poletto, Rev. Sci. Instrum. 74, 2992 (2003).

${ }^{4}$ R. P. Wayne, Chemistry of Atmospheres, 3rd ed. (Oxford University Press, New York, 2000).

${ }^{5}$ B. M. Smirnov, Physics of Ionized Gases (John Wiley and Sons, New York, 2001).

${ }^{6} \mathrm{O}$. Christiansen, C. Hättig, and P. Jørgensen, Int. J. Quantum Chem. 68, 1 (1998).

${ }^{7}$ T. Helgaker, S. Coriani, P. Jørgensen, K. Kristensen, J. Olsen, and K. Ruud, Chem. Rev. 112, 543 (2012).

${ }^{8}$ P. W. Langhoff, Chem. Phys. Lett. 22, 60 (1973).

${ }^{9}$ P. W. Langhoff and C. Corcoran, J. Chem. Phys. 61, 146 (1974).

${ }^{10}$ P. W. Langhoff, C. Corcoran, J. Sims, F. Weinhold, and R. Glover, Phys. Rev. A 14(3), 1042 (1976)

${ }^{11}$ K. Gokhberg, V. Vysotskiy, L. S. Cederbaum, L. Storchi, F. Tarantelli, and V. Averbukh, J. Chem. Phys. 130, 064104 (2009).

${ }^{12}$ S. Kopelke, K. Gokhberg, L. S. Cederbaum, F. Tarantelli, and V. Averbukh, J. Chem. Phys. 134, 024106 (2011).

${ }^{13}$ V. Averbukh and L. S. Cederbaum, J. Chem. Phys. 123, 204107 (2005).

${ }^{14}$ S. Kopelke, K. Gokhberg, V. Averbukh, F. Tarantelli, and L. S. Cederbaum, J. Chem. Phys. 134, 094107 (2011).

${ }^{15} \mathrm{~S}$. Coriani, O. Christiansen, T. Fransson, and P. Norman, Phys. Rev. A 85, 022507 (2012).

${ }^{16} \mathrm{~S}$. Coriani, T. Fransson, O. Christiansen, and P. Norman, J. Chem. Theory Comput. 8, 1616 (2012).

${ }^{17}$ J. Berkowitz, Atomic and Molecular Photoabsorption: Absolute Total Cross Sections (Academic Press, London, 2002).

${ }^{18}$ G. H. Golub and C. F. van Loan, Matrix Computations, 2nd ed. (The Johns Hopkins University Press, Baltimore, 1989). 
${ }^{19}$ O. Christiansen, H. Koch, and P. Jørgensen, Chem. Phys. Lett. 243, 409 (1995).

${ }^{20}$ B. N. Parlett, The Symmetric Eigenvalue Matrix Computations, 2 nd ed. (Prentice-Hall, Englewood Cliffs, NJ, 1980).

${ }^{21}$ Z. Bai, J. Demmel, J. Dongarra, A. Ruhe, and H. van der Vorst, eds. Templates for the Solution of Algebraic Eigenvalue Problems: A Practical Guide (SIAM, Philadelphia, 2000).

${ }^{22}$ I. H. Godtliebsen and O. Christiansen, Phys. Chem. Chem. Phys. 15, 10035-10048 (2013).

${ }^{23}$ F. Muller-Plathe and G. H. Diercksen, "Molecular photoionisation cross sections by moment theory. An introduction," in Electronic Structure of Atoms, Molecules and Solids. Proceedings of the II Escola Brasileira de Estructure Eletronica, Olinda, Brazil, 17-22 July 1989, edited by S. Canuto, J. D’Albuquerque e Castro, and F. J. Paixao (Olinda, Brazil, 1990), pp. 1-29.

${ }^{24}$ R. K. Nesbet, Phys. Rev. A 14, 1065 (1976).

${ }^{25}$ C. Hättig, O. Christiansen, and P. Jørgensen, J. Chem. Phys. 107, 10592 (1997)

${ }^{26}$ DALTON, A Molecular Electronic Structure Program, 2011. Release Dalton2011, see http://daltonprogram.org/.

${ }^{27}$ K. Kaufmann, W. Baumeister, and M. Jungen, J. Phys. B: At. Mol. Opt. Phys. 22, 2223 (1989).
${ }^{28}$ T. Helgaker, P. Jørgensen, and J. Olsen, Molecular Electronic-Structure Theory (John Wiley and Sons, Chichester, 2000).

${ }^{29}$ M. Stener, P. Decleva, and A. Lisini, J. Electron Spectrosc. Relat. Phenom. 74, 29 (1995).

${ }^{30}$ J. A. R. Samson and W. C. Stolte, J. Electron Spectrosc. Relat. Phenom. 123, 265 (2002).

${ }^{31}$ J. A. R. Samson and G. N. Haddad, J. Opt. Soc. Am. B 11, 277 (1994).

${ }^{32}$ F. Carnovale, R. Tseng, and C. E. Brion, J. Phys. B 14, 4771 (1981).

${ }^{33}$ G. N. Haddad and J. A. R. Samson, J. Chem. Phys. 84, 6623 (1986).

${ }^{34}$ W. Chan, G. Cooper, and C. Brion, Chem. Phys. 178, 387 (1993).

${ }^{35}$ G. R. Burton, W. F. Chan, G. Cooper, and C. Brion, Chem. Phys. 177, 217 (1993).

${ }^{36}$ W. Chan, G. Cooper, and C. Brion, Chem. Phys. 170, 123 (1993).

${ }^{37}$ W. Chan, G. Cooper, and C. Brion, Chem. Phys. 178, 401 (1993).

${ }^{38}$ N. Padial, G. Csanak, B. V. McKoy, and P. W. Langhoff, J. Chem. Phys. 69, 2992 (1978).

${ }^{39}$ G. H. F. Diercksen, W. P. Kraemer, T. N. Rescigno, C. F. Bender, B. V. McKoy, S. R. Langhoff, and P. W. Langhoff, J. Chem. Phys. 76, 1043 (1982).

${ }^{40}$ C. Hättig and F. Weigend, J. Chem. Phys. 113(13), 5154 (2000).

${ }^{41}$ C. Hättig and K. Hald, Phys. Chem. Chem. Phys. 4, 2111 (2002). 